\title{
Community-Based Care of the Elderly in Rural Japan: A Review of Nurse-Led Interventions and Experiences
}

\author{
Yoshiyuki Nagaya • Angela Dawson
}

\begin{abstract}
Nurses play a critical role in delivering care to elderly people at primary health care level but there is no synthesis of research knowledge to guide community nursing practice in Japan. This review aims to identify nurse-led interventions that have been found to improve elder health at village level; the barriers and constraints that service providers face when delivering care to the elderly; and the experiences of elderly people and their caregivers. The electronic databases such as MEDLINE, CINAHL and Google Scholar were searched to retrieve peerreviewed primary research literature. A narrative synthesis of the findings sections of the papers was applied to identify key themes. These themes are: socioculturally appropriate care; health improvements; barriers and constraints to care delivery and; experience of the elderly and families. Seven papers were included in the review. The synthesis identified that nurse-led health care for the elderly in rural Japan can be effective when it is targeted and culturally sensitive. The studies highlight a number of barriers to the provision of care. There is a need for further research to examine the issues affecting access to rural nursing care including health system factors, as well as the needs of the elderly and families themselves. Such studies will better inform the delivery of programs, reduce inequity and provide socioculturally appropriate care to improve the well-being of the elderly.
\end{abstract}

\section{Background}

Community Health Nursing in Japan A critical part of the Japanese health system is the nursing profession, and more importantly community health nursing. The concept of a visiting or community nurse can be traced back to the late 1880s when nurses provided visiting nursing services, on a volunteer basis, for mothers, children and disaster survivors [1]. The visiting nurse system was broadened in the 1960s when bed-bound elderly suffering from cerebral diseases became a significant social problem in Japan [2, 3]. By 1984, about 8 $\%$ of all Japanese hospitals were providing community nursing services. By 1999, the 
percentage had reached almost half of all Japanese hospitals. In addition to hospitals, in the 1980s and 1990s, the visiting nursing system also involved being general clinics [4].

There are different nurses who play a role in community nursing in Japan. Public health nurses (PHNs) are more senior than other types of community nurses such as registered nurses and play a significant role in health promotion, disease prevention and community development. They also manage health programs and contribute to policy formulation $[4,5]$. PHNs educate and lead community health nurses, they provide guidance on complex issues and often serve as supervisors for the work performedby community health nurses. To be registered as a PHNa high level tertiary entrance score is required to enter the pre-requisite nursing education [6]. The role of PHNs increased, when long-term care insurance and initiatives were introduced to contain health care spending by the Government [4].

\section{Crisis in Elderly}

Care Japan is one of the most densely populated countries in the world with 127.3 million people in 2013 [7]. Japan's population growth is extremely low and forecasted to be negative in the future. Japan is also the world's most rapidly ageing society. Labor shortages are evident in elderly care in Japan. Calls continue to recruit foreign workers to address the shortage of low-paid, low-skilled home healthcare helpers [8]. The provision of care to the elderly has also been affected by the increased participation of women in the workforce. By 1998, more than $50 \%$ of working-age women in Japan were employed. As women are often the carers of elderly parents, women are finding it increasingly difficult to both participate in the labor market and provide in-home care for elderly family members.

Growing urbanization for employment has led to smaller nuclear families who are no longer capable of providing support to the elderly who may still reside in rural areas [9]. The culture of care for the elderly has also changed. Individuals in their forties and fifties no longer feel responsible for looking after elderly family members [10]. The elderly do not always like being cared for by their younger relatives, preferring instead to be cared for by health professionals or to look after themselves [11]. However, in traditional Japanese culture, it is uncommon for individuals to rely on others outside the family for care as the elderly prefer to remain independent. This cultural phenomena means that many elderly persons, even if Government services are available, will be cared for by their relatives [12]. 
Health Policies Affecting Home Based Elderly Care

Japan is making efforts to address the crisis in elderly care by extending the availability of its long-term care insurance. Japan has started offering subsidised public services at the local level as part of its Gold Plan, a 10-year plan enacted in 1989 to support home health care instead of institutionalization. In 2000, the Gold Plan added the provision of visiting nursing services to its insurance coverage [13]. To receive community health services from nurses in their homes, or at the visiting nursing station under the long-term care insurance scheme, individuals require an 'order' from a primary care physician [4]. After referral, the visiting nursing station will regularly report to the primary care physician on the progress of the patient. The visiting nursing station will also engage with the manager of home-care on an ongoing basis. This provider collaboration ensures that the patient's care is monitored and regularly assessed (Fig. 1).

Fig. 1 Application for visiting nursing services and start of service (Japan Visiting Nursing Foundation 2006)

\section{In case of using the long-term care insurance system}

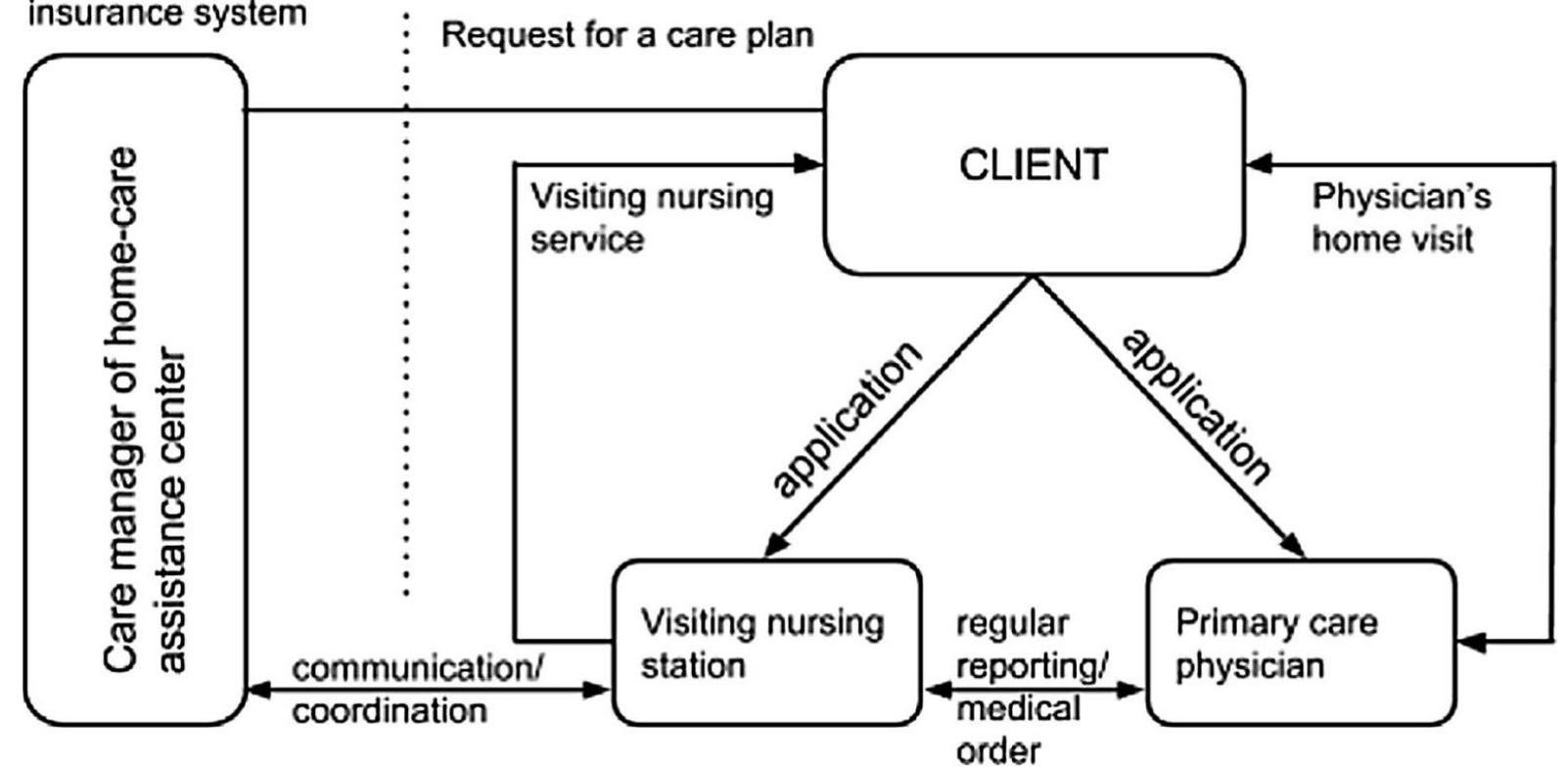

Despite these important efforts to address the care of the elderly at community level in Japan there is no synthesis of evidence to guide the practice of visiting nurses in this unique context. Therefore, the aim of this paper is to identify nurse-led interventions that have been 
found to improve elder care at village level; the barriers and constraints that nurses face when delivering care to the elderly; and the experiences of elderly, their families and caregivers.

\section{Methodology}

The review involved a search of the databases MEDLINE and CINAHL, the search engine Google Scholar and hand searching of the reference lists of retrieved papers to recover primary research peer-reviewed literature in both English and Japanese, published between 2000 and 2013. Table 1 outlines the number of items initially retrieved while Fig. 2 demonstrates the screening process according to the PRISMA guidelines [14]. Due to the varied nature of the methods found in the studies retrieved, a narrative synthesis methodology [15] was considered appropriate.

The search terms applied were: 'community nursing' or 'visiting nurse' or 'home visiting' and 'elderly' and 'rural' and 'Japan'. The qualitative studies included in this review were appraised by applying the Critical Appraisal Skills Programme qualitative research [16] and the non-experimental studies were assessed using Law's Critical Review Form [17]. Both authors of this paper discussed the relevancy of each paper to the review questions and agreed which papers should be included. The findings sections of each paper were analysed thematically and patterns drawn across each study. The themes and interpretation was carried out by both authors.

\begin{tabular}{lr}
\hline Database & $\begin{array}{r}\text { Records } \\
\text { retrieved }\end{array}$ \\
\cline { 2 - 2 } MEDLINE & 11 \\
CINAHL & 4 \\
Google Scholar & 16,200 \\
Hand searching from reference lists of retrieved items & 19 \\
& 16,234 \\
\hline
\end{tabular}

Fig. 2 Literature review process 


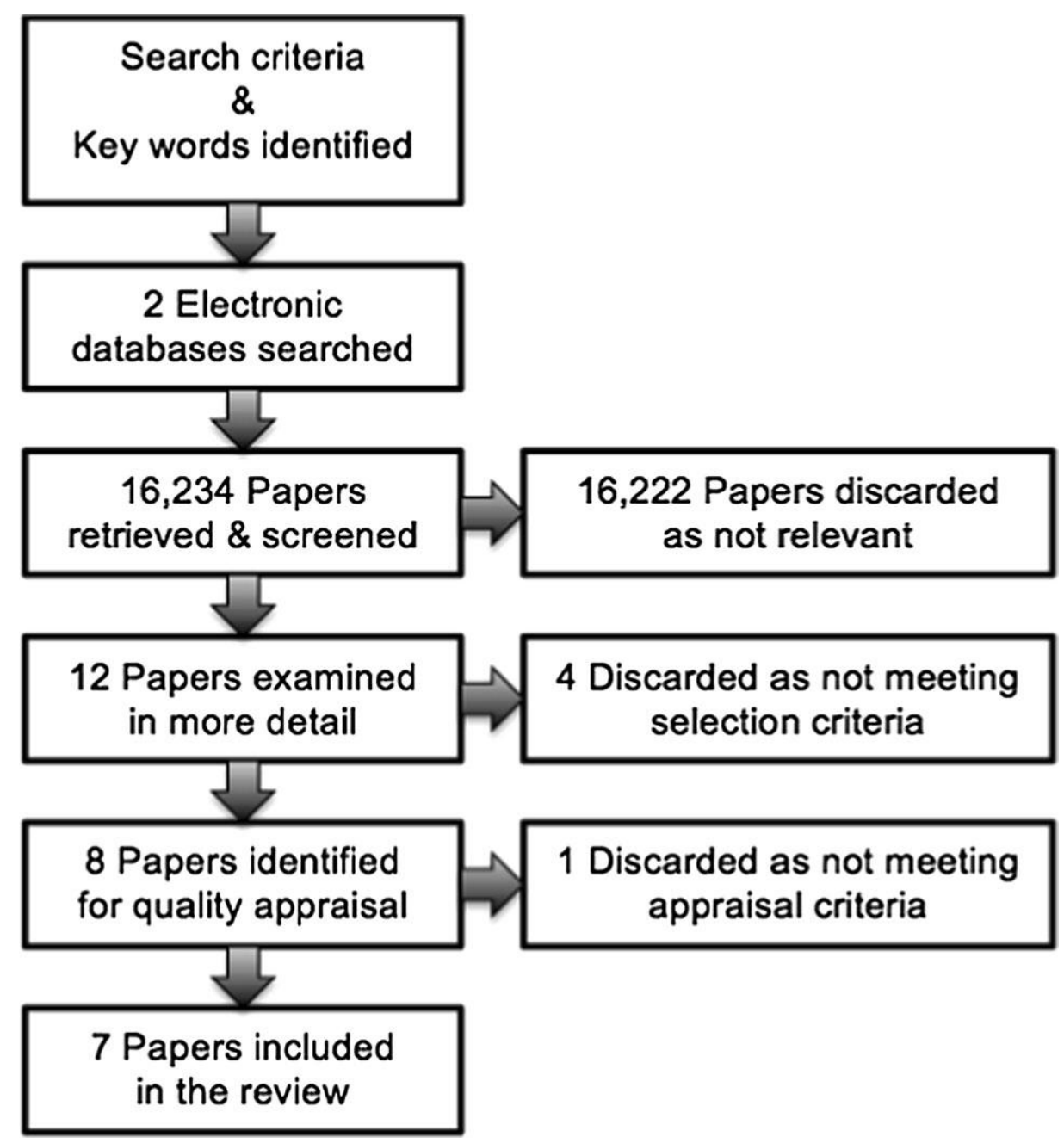

Findings

A summary of the studies included in this review is shown in Table 2. The review included seven papers. Two of the papers are qualitative studies, four papers are quantitative and one of the paper employed mixed methods. Two papers did not identify the particular rural area studied in Japan. Five papers involved research in two of Japan's four islands (northern, central and southeast part of Honshu, and northern and southern part of Kyushu, see Fig. 3). The findings in this project are presented according to the key themes: Culturally appropriate care; health improvements; barriers and constraints to care delivery; experience of the elderly and families.

Socioculturally Appropriate Nurse-Led Interventions for the Elderly at Village Level Elderly care at the rural level is most effective when nurses take into account the dietary practices of the elderly, relationships with neighbors, and residents' values and beliefs [18]. A 
number of approaches to the provision of culturally sensitive nursing care including showing an interest in, and respect for the local culture and farming practices and developing trust with elderly people [18]. The authors describe PHNs strategies that involved engaging in dialogue with the elderly about modifications in farming and eating habits that resulted in behavioral change. At first, PHNs acknowledged the views of local elderly, but in time, by developing trust with them they began to challenge the need for the excessive consumption of particular types of foods (e.g. corn and sushi) that have a high sugar content.

Another PHN approach involved nurses raising elders' awareness of health risks using familiar examples including demonstrating the difference in taste between different types of salted and non-salted food in order to reduce their salt intake [18]. Also, PHNs attempts to accept and understand the inconsistency between local culture and healthy practice were well received by the elderly. This was illustrated using the case of a PHN who pointed out to an elderly women that despite the need for her to reduce the amount of sugar taken in a roll sushi, the PHN understood that the practice had come from a time in the past where sugar had been a special treat. PHNs in the study were found to be concerned about overwork among the elderly. The strong work ethic of many rural Japanese, together with inherent feelings of guilt in neglecting the fields, was often causing health problems. PHNs encouraged the elderly to work early in the morning and late in the afternoon, to take regular breaks and enjoy their later years without any feelings of guilt. PHNs sought to adjust their healthpromotion efforts to fit their local culture of the elderly. By understanding the local behaviors, needs and traditions, PHNs have been able to find innovative ways to promote health and prevent health problems [18].

\section{Health Impact on Suicidal Rates}

Two studies in the review assessed the effectiveness of community based interventions to address depression and suicide among the elderly in two towns in rural Japan [19, 20]. This research found that education interventions delivered by PHNs targeting suicide using depression management with non-psychiatric, primary health care lowered suicide rates for elderly women by $70 \%$ or more in comparison to men. Programs comprised of three main elements: depression detection (using firstly self-reporting and secondly annual assessments by PHNs), workshops 3-4 times annually, and social activities and exercise on a monthly basis. During workshops, local PHNs promoted depression awareness and management of 
suicide risk. The reasons for these results need to be investigated further to determine what measures will prove effective to reduce suicide rates for both sexes. To some extent, it may be that the results are merely reflective of the small population the subject of the study. It may therefore be difficult to generalize the results.

\section{Barriers and Constraints Facing the Delivery of Elderly Health Care}

Barriers and constraints to the provision of care were noted by two studies included in the review [21, 22]. Some inequities between use and access to home nursing care in situations where individuals have caregivers and where elderly may live on their own [21]. Where elderly had family or home help, nurses reported that they could better facilitate the provision of visiting nursing services than in contexts where individuals live alone. Staying with relatives or homehelp service use and requiring low levels of care was found to be correlated with better access to visiting nursing services. Higher usage of home-help services and those of visiting nursing services were found among elderly study participants who lived on their own. Such services will only be used, however, if they are affordable.

In contexts where higher care levels are required, income levels or a lack of regular hospital visits before joining long-term health care insurance programs were found to be the main factors limiting home visit nursing services use. A care plan was found to reduce the use of visiting nursing services for lower care level patients, but not for higher care patients. However, visiting nursing services are not commonly recommended to patients from lower level care groups. Low income earners with high care needs may not be using visiting nursing services as a result of the effect of the $10 \%$ user copayment [21].

A lack of screening was found to be a barrier to the delivery of community nursing services to the elderly [22]. Some patients (e.g. patients suffering from dementia) might be still in need of greater visiting nurse services [22]. The research shows that the higher the level of care needed, the more likely patients are to rely upon and require nurse services. Visiting nurse services use was found to increase as the cost fell. The impact of care needs and costs on the degree of use of visiting nurses services needs to be more fully examined before any conclusions can be reached [22]. 
Certain difficulties have been found with the visiting nursing system in rural Japan. This includes travel costs and the length of journey to remote areas, as well as the challenge of transporting equipment such as portable baths [23].

Experiences of Elderly and Caregivers

Japanese families who were concerned about what others think or say about seeking assistance for the care of their elderly relatives were less likely to use public services than those that were not concerned about what others thought [24]. Those caring for the elderly with severe dependency in activities of daily life were three times more likely to use Government care. Caregivers may decide to use services based on the need for help, rather than whether or not it is available.

\section{Discussion}

This review has identified the importance of socioculturally appropriate and targeted community nursing strategies Effective health and screening was found in three studies to change lifestyle behaviors and lower suicide rates among specific elderly populations in rural contexts in Japan when nurses engaged elderly people and took time to understand their needs. This finding is consistent with other research. Home-based nursing health promotion for older people in countries other than Japan showed that specifically targeted approaches often prove more successful than generalist programs [25]. In all countries where community nursing has been adopted, the importance of greater patient contact has been highlighted. General contact may not allow the nurse to fully understand the patient and provide the best care. Patient follow up and taking time to develop trust with elderly people can provide nurses with a comprehensive understanding of their patient's circumstances which may be critical to improving health outcomes [26].

The two studies in this review concurred with the findings of other research that demonstrated a decrease in suicide rates after the delivery of community-based health promotion programs by a variety of professionals in Japanese rural towns [19, 27]. Visits by PHNs to home bound elderly, to prevent feelings of loneliness (particularly in winter) may also be of assistance in reducing suicidal rates [27]. It is not clear why the community 
intervention program was more effective preventing suicide in elderly females, but not males [19]. This has been identified elsewhere [28] suggesting that the factors that may lead to depression among elderly men including involuntary retirement, pain, dependency, daily hassles, sleep problems, loss of driver's license, bereavement and alcohol misuse should be taken into consideration in the design of health promotion programs. Involvement by the elderly in social and educational activities can effectively reduce one of the more common risk factors of suicide, being depression [20]. High levels of abuse toward the elderly are found in many communities [29] which may have an impact upon suicidal rates. Depression is a significant risk factor associated with elder abuse and neglect among community-based Chinese elderly [30]. This highlights the need for health professionals to take particular note of depression along with associated factors including being widowed/divorced/single/separated, having a labor intensive job, having a physical disability, and living alone [31]. Multidisciplinary approaches may also be required to manage such patients [32].

This narrative synthesis also shows that factors such as: the living arrangements of the elderly, whether on their own or with family members; the views of family members; the use of home help; economic status; level of care needed and; the level of community screening undertaken affected the use of home nursing visiting services. However, there is a paucity of evidence available from a range of rural Japanese settings to best guide community nursing for older people. Few high quality studies were retrieved for this review. Despite this, the review has highlighted particular areas that require further investigation in order to deliver the most appropriate, accessible and quality nursing care to the elderly in rural contexts. This includes issues related to the equitable provision of care, the relationship between care provided by families at home and nursing care and particular challenges to health care delivery in rural community settings.

\section{Equity in Home Visiting Nursing Services}

Several areas were indicated when inequity may affect access to nurse visiting services for those elderly who need it most [21]. Elderly who live alone or utilize non-government services may be more vulnerable than those who have caregivers who are family members or home help. Home visit nursing services' use fell where the individual either lived alone, used a corporate type of care management agency or relied on the services of a non-medical care 
management agency to create a service plan [21]. Access to home visit nursing services was also found to be better facilitated when the elderly have family caregivers or home help, compared to those who depend on care management agencies alone. In addition, the findings also suggest that Japan's long-term care insurance system may be limiting the use of visiting nursing services by low-income earners with higher care needs. Nurses have a key role to play in addressing health inequities. This can involve targeting screening for vulnerable patients, working in partnership with other members of health and social care organisations, engaging local people and groups, in public health awareness and action and utilising public health evidence in everyday practice, not just evidence for treating illness [33]. However, the provision of universal health care must be considered within a health system approach [34] where financing and governance issues are considered alongside workforce ones. To encourage equity in the delivery of health services, Japanese policy makers will need to reconsider the present provision of home-visit nursing services.

\section{Family Home Care and Nursing Care}

Some Japanese families did not seek health assistance from community nurses, due to public shame about not fulfilling their family duties to provide services to the elderly [24]. The notion of care for the elderly as a family duty is still widespread in rural Japan and has been associated with older persons' well-being [35]. This practice is evident in other cultures such as the Chinese concept of filial piety. Chinese tradition involves the provision of physical care for ones ageing parents at home and has been adopted into law requiring all adult children take care of their ageing parents with the government only providing care for the childless elderly [36]. This may not be feasible in Japan and other approaches such as advocacy and awareness campaigns may be more appropriate to encourage family engagement in elderly care, increase the use of health services and reduce the shame factor associated with the seeking of health assistance. Raising the awareness of health professionals may also be necessary $[37,38]$. The stigma attached to young individuals who do not provide care to the elderly in Japan may change over time, as has been found in China where there has been a shift in attitudes concerning the involvement of professionals in elder care from stigma to privilege [39].

The elderly may in some cases prefer community nursing care than family provided care at their home. The experience of the elderly living with extended family in their own home 
provides insight into tensions that affect care decisions [40]. An example of an elderly women residing in her son's marital household is a case in point. In this example the woman was embarrassed by living with her daughter-in-law and disappointed and humiliated because she did not receive care from her own children. This led to choosing to move to a nursing home [40].

Challenges to Delivering the Visiting Nursing System in rural Areas

The challenges of providing care to the rural Japanese elderly have been examined in a number of reports [41] and are in line with a paper included in this review [23]. Transportation costs and the length of the journey to the homes of the elderly as making it difficult for nurses to access remote areas of Japan [23]. These difficulties, along with the current workforce shortages in elderly care may affect the recruitment and retention of nurses to rural areas as experienced in countries such as Australia [42]. Japan may need to closely examine the factors found elsewhere to attract and retain nurses in such contexts $[43,44]$ and design appropriate workforce interventions such as education and training and financial incentives.

Based upon the findings of this review a number of issues may need to be considered in the design and implementation of community nursing initiatives in rural Japan. These should include the following:

1. socioculturally appropriate and tailored care health promotion that takes into consideration the lifestyles of the elderly, their needs and the family shame associated with carers seeking help;

2. health promotion efforts should include nutrition and depression;

3. elderly people living alone should be targeted for screening and health education;

4. nurses should engage with family members and other professionals to ensure collaborative approaches to

care and;

5. workforce interventions should be considered to attract and retain nurses into the visiting nursing program.

\section{Conclusion}


Elderly care is critical to Japanese society. A rapidly ageing population, low growth rates and economic recession have cast doubt on the Japanese Government's ability to care for its citizens. Shortages in carers will place strain on health care in Japan, particularly with respect to the elderly. The delivery of elderly health care, particularly in rural areas, in Japan, is not without difficulties. Longstanding traditions, eating habits and lifestyle patterns make it difficult for nurses to change the habits of local Japanese. Nurses have to adapt to local customs and culture to ensure that changes are made by locals. This review has found that socioculturally sensitive approaches are needed to deliver appropriate care. The difficulties confronted in Japan are by no means unique. Greater numbers of elderly living on their own in remote, often inaccessible areas appears to be, to a varying degree, a global problem. The encouragement of family care and the recognition of the benefits of community health professionals are important. Health systems need to be responsive and ensure that policies reduces barriers to elderly access. For Japanese community nursing, a relationship of dialogue, trust and cultural sensitivity is beginning to be acknowledged as a recipe for improved health of patients. More research into nurse-led interventions at community level is necessary to ensure universal access and improved health outcomes for the rural elderly

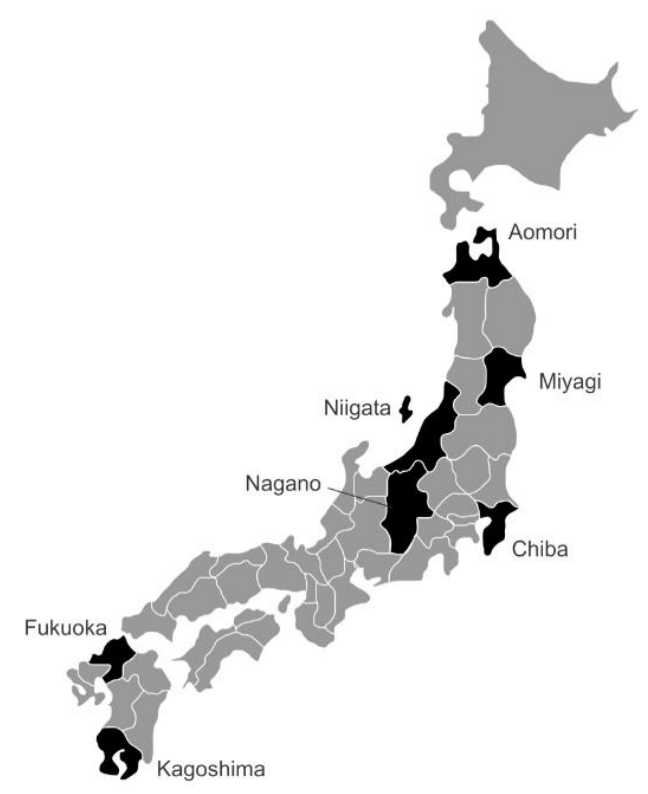

Fig. 3 Japanese prefectures where studies were undertaken.

Note Not including papers examining anonymous prefecture 


\section{Fusho et al. Prefecture not specified}

(2006)

\section{Kashiwagi et al. Kagoshima (southern part of Kyushu, \\ (2013) surrounded by the sea on 3 sides)}

Marutani \& $\quad 3$ rural communities location not specified
Miyazaki

(2010)

Nagata

et al.
(2013)

Oyama et al.

(2006a)

Oyama et al.

(2006b)

Miyagi (northeastern part of Honshu, facing the Pacific Ocean), Chiba (southeast part of Honshu, facing the Pacific Ocean), Nagano (central part of Honshu), and Fukuoka (northern coast of Kyushu)

Niigata (center of Honshu, facing the Sea of Japan)

Aomori (northern part of Honshu, surrounded by the sea on 3 sides)
Mixed methods. Descriptive quantitative design using validated instruments. A comparison of users and non-users of nursing services undertaken for a number of variables. Qualitative interviews with caregivers.

Descriptive qualitative survey design.

Descriptive quantitative survey design. examined one town during study and partook in other health-care programs in town. services after individual left hospital. implemented for 10 years in Matsudai town).
Descriptive qualitative study. Authors observed health counselling sessions performed by PHNs with elderly farmers. After each session, PHNs were interviewed on their views on dialogue. Each researcher

Quantitative cross-sectional correlation study. Nursing staff gathered patients' information and examined needs not met for visiting nurse

Descriptive qualitative study using depression screening tool with followup and health education using primary care \& public health nursing, was

Quantitative study. Suicide data sourced from potential registration of suicide events at Hachinohe Public Health Center. Register diagnoses came from International Classification of Diseases- 9 , in which confirme suicides and potential suicides were addressed together.
1586 individuals in town were over 65,7 caregivers

21 providers at $(70.0 \%)$ facilities providing community nursing (13 facilities)

1276 users of community-based services covered by long-term care insurance were examined

22 men \& 19 women with a mean age

To assess whether views of strangers impact use of Government health care by elderly

To examine practical implementation of rural community nursing initiatives

To examine predictors of home visit nursing services use covered by long-term care insurance using claim information \& surveys performed on insurers of long term care insurance in six rural towns

of 68 years (range: $65-73$ years)

To assess methods used by PHNs to undertake culturally sensitive health counseling for elderly farmers to encourage them to adopt healthy behaviours

1594 sample patients aged 65 years or over discharged from general units in hospitals in 4 prefectures after a stay hospitals in 4 prefectur 14 days or longer.
of

To analyse whether certain home care patients have needs not met by visiting nurse services (especially after leaving hospital)

Matsudai town: population 6,015; suicide rate per $10^{5}$ (65-year-olds) fo males $290.6, \&$ for females 361.3

To evaluate outcomes of a community-based program to prevent suicide among the elderly using a quasiexperimental design with a neighboring reference group

Kawanishi town: population 9,425 elderly suicide rate for males 212.2 females 151.9

43 (7 males \& 36 females) in 1993 1998

106 (16 males \& 90 females) in 1999 2004
To evaluate results of community-based program to reduce suicide rates of elderly ( $\geq 65$ years old) using a quasi-experimental design with two neighboring references 
Arai, Y., Sugiura, M., Miura, H., Washio, M., \& Kudo, K. (2000). Undue concern for others' opinions deters caregivers of impaired elderly from using public services in rural Japan. International Journal of Geriatric Psychiatry, 15, 961-968.

Bessho, Y., Hosoya, T., Hasegawa, M., Yoshida, Y., \& Matsuki, M. (2004). Home nursing care to achieve the expected status for the elderly with cerebro-vascular diseases. Journal of Japanese Society of Nursing Research, 27(5), 65-71.

Enguidanos, S., Gibbs, N., Simmons, W., Savoni, K., Jamison, P., Hackstaff, L., et al. (2003). Kaiser permanente community partners project: Improving geriatric care management practices. Journal of the American Geriatric Society, 51(5), 710-714.

Fusho, S., Matsuyama, Y., Mori, H., \& Matsushita, M. (2006). Role of visit nursing from the hospital and clinic in a depopulated area in a prefecture. Gifu College of Nursing, 6(2), 43-50.

Hooyman, N., \& Kiyak, A. (2011). Social gerontology: A multidisciplinary perspective. (9th ed.). London: Pearson.

Japan Visiting Nursing Foundation. (2006). Visiting Nursing System in Japan. JVNF. Tokyo. Retrieved August 16, 2013, from http://www.jvnf.or.jp/homon/0201-2/

Kashiwagi, M., Tamiya, N., Sato, M., \& Yano, E. (2013). Factors associated with the use of home-visit nursing services covered by the long-term care insurance in rural Japan: A crosssectional study. BioMed Centra, doi:10.1186/1471-2318-13-1.

Liebel, D., Friedman, B., Watson, N., \& Powers, B. (2009). Review of nurse home visiting interventions for community-dwelling older persons with existing disability. Medical Care Research and Review, 66(2), 119-146.

Long Term Care Insurance Act. (1997). Tokyo.

Markle-Reid, M., Browne, G., Weir, R., Gafni, A., Roberts, J., \& Henderson, S. (2006). The effectiveness and efficiency of home-based nursing health promotion for older people: A review of the literature. Medical Care Research and Review, 63(5), 531-569.

Marutani, M., \& Miyazaki, M. (2010). Culturally sensitive health counseling to prevent lifestyle-related diseases in Japan. Nursing and Health Science, 12, 392-398.

Matsuda, M. (2007). Jikei byouin hasyutsu kangohu-kou [Community Nursing theory of Jikei hospital]. Jikei University. Tokyo.

Ministry of Health, Labour and Welfare. (n.d.). Syuyou shiin-betsu so-shibou-ritsu no joukyou [Situation of major cause-specific crude death rate], MHLW, Tokyo, Retrieved August 23, 2013, from http://www.mhlw.go.jp/toukei/saikin/hw/jinkou/tokusyu/sinno05/2.html

Ministry of Internal Affairs and Communications, Statistic Bureau. (2013). Jinkou-suikei [Population estimates]. MIACSB. Tokyo. Retrieved August 23, 2013, from http://www.stat.go.jp/data/jinsui/new.htm 
Morgan, C. (2001). Demographic crisis in Japan: Why Japan might open its doors to foreign home health-care aides. Pacific Rim Law \& Policy Journal Association, 10(3), 749-779.

Motohashi, Y., Kaneko, Y., Sasaki, H., \& Yamaji, M. (2007). A decrease in suicide rates in Japanese rural towns after community-based intervention by the health promotion approach, Suicide and Life-Threatening Behavior, 37(5), 593-599.

Murashima, S., Hatono, Y., Whyte, N. \& Asahara, K. (1999). Public health nursing in Japan: New opportunities for health promotion. Public Health Nursing, 16(2), 133-139.

Nagata, S., Taguchi, A., Naruse, T., Kuwahara, Y., \& Murashima, S. (2013). Unmet needs for visiting nurse services among older people after hospital discharge and related factors in Japan: Cross-sectional survey. Japan Journal of Nursing Science, doi:10.1111/jjns.12012.

Nicolaides-Bouman, A., Van Rossum, E., Kempen, G., \& Knipschild, P. (2004). Effects of home visits by home nurses to elderly people with health problems: Design of a randomized clinical trial in the Netherlands. BioMed Central Health Services Research, 4(5), 4-35.

Oyama, H., Goto, M., Fujita, M., Shibuya, H., \& Sakashita, T. (2006a). Preventing elderly suicide through primary care by community-based screening for depression in rural Japan. Crisis: The Journal of Crisis Intervention and Suicide Prevention, 27(2), 58-65.

Oyama, H., Ono, Y., Watanabe, N., Tanaka, E., Kudoh, S., Sakashita, T., et al. (2006b). Local community intervention through depression screening and group activity for elderly suicide prevention. Psychiatry and Clinical Neurosciences, 60, 110-114.

Public Health Nurses, Midwives and Nurses Act. (1948). Tokyo.

Van Hout, H., Nijpels, G., Van Marwijk, H., Jansen, A., Van't Veer, P., Tybout, W., et al. (2005). Design and pilot results of a single blind randomized controlled trial of systematic demand-led home visits by nurses to frail elderly persons in primary care. BioMed Central Health Services Research, 5(11), 1-19.

Wailerdsak, N. (2009). Foreign workers from South-East Asia: Issues regarding acceptance of nurses and caregivers under the economic partnership agreements. Toyo-bunka Kenkyusho-kiyou, 155, 129-171.

Wu, Y. (2004). The care of the elderly in Japan, Caring for Japan's elderly, 39, 224, London: RoutledgeCurzon. 